\title{
Robust Stability and Stabilization for Singular Time-Delay Systems with Linear Fractional Uncertainties: A Strict LMI Approach
}

\author{
Jinxing Lin ${ }^{1,2}$ and Lina Rong \\ ${ }^{1}$ College of Automation, Nanjing University of Posts and Telecommunications, Nanjing 210023, China \\ ${ }^{2}$ Key Laboratory of Measurement and Control of Complex Systems of Engineering, Ministry of Education, Southeast University, \\ Nanjing 210096, China
}

Correspondence should be addressed to Jinxing Lin; jxlin2004@126.com

Received 21 August 2013; Accepted 24 September 2013

Academic Editor: Tao Li

Copyright (C) 2013 J. Lin and L. Rong. This is an open access article distributed under the Creative Commons Attribution License, which permits unrestricted use, distribution, and reproduction in any medium, provided the original work is properly cited.

\begin{abstract}
This paper is concerned with the problems of delay-dependent robust stability and stabilization for a class of continuous singular systems with time-varying delay in range and parametric uncertainties. The parametric uncertainties are assumed to be of a linear fractional form, which includes the norm bounded uncertainty as a special case and can describe a class of rational nonlinearities. In terms of strict linear matrix inequalities (LMIs), delay-range-dependent robust stability criteria for the unforced system are presented. Moreover, a strict LMI design approach is developed such that, when the LMI is feasible, a desired state feedback stabilizing controller can be constructed, which guarantees that, for all admissible uncertainties, the closed-loop dynamics will be regular, impulse free, and robustly asymptotically stable. Numerical examples are provided to demonstrate the effectiveness of the proposed methods.
\end{abstract}

\section{Introduction}

Singular time-delay systems, which are known as descriptor time-delay systems, implicit time-delay systems, or generalized differential-difference equations, often appear in various engineering systems, for example, aircraft attitude control, flexible arm control of robots, large-scale electric network control, chemical engineering systems, lossless transmission lines, and so forth $[1,2]$. Since singular time-delay systems are matrix delay differential equations coupled with matrix difference equations, the study for such systems is much more complicated than that for standard state-space time-delay systems. Recently, a great deal of attention has been devoted to the study of such more general class of delay systems; see [3-27].

The existing stability criteria for singular time-delay systems can be classified into two types: delay independent [35] and delay dependent [6-10]. Generally, delay-dependent conditions are less conservative than the delay-dependent ones, especially when the time delay is small. To obtain delaydependent conditions, many efforts have been made in the literature, among which the model transformation and bounding technology for cross-terms [8-10] are often used. However, it is known that the bounding technology and the model transformation are the main source of conservation [28]. Recently, some improved stability conditions with less conservatism have been provided by utilizing the free weighting matrix method [11-13], the integral inequality [14], and the delay decomposition approach [15-17], in which neither the bounding technology nor model transformation is involved. However, these conditions in [6-17] were established under the assumption that the delay was time invariant. For the continuous singular systems with time-varying delay, Yue and Han investigated the delay-dependent stability condition by introducing the free weighting matrices [18]. In [19], a delay-dependent stability condition was presented by using the integral inequality method. But the range of the time-varying delay considered in $[18,19]$ is from 0 to an upper bound. In practice, a time-varying interval delay is often encountered; that is, the range of delay varies in an interval for which the lower bound is not restricted to 0 . In this case, the stability criteria in $[18,19]$ are conservative 
because they do not take into account the information of the lower bound of delay. Moreover, when estimating the upper bound of the derivative of Lyapunov functional, some useful terms are ignored in $[18,19]$. More recently, continuous singular systems with time-varying delay in a range have been extensively studied; see, for example, [20-27] and references therein.

On the other hand, in recent years, more and more attention has been devoted to derive strict LMI conditions for stability analysis and controller design; see, for example [29, 30] and references therein. The strict LMI conditions, that is, definite LMIs without equality constraints, are highly tractable and reliable when checked by some recently developed algorithms for solving LMIs [31]. However, it should be pointed that the stability conditions derived in [20-27] are formulated in terms of nonstrict LMIs, whose solutions are difficult to calculate since equality constraints are often fragile and usually not met perfectly. Furthermore, up to now, to the best of the authors' knowledge, for a continuous uncertain singular system with a time-varying interval delay, the problems of robust stability, stabilization, and feedback control have not been fully investigated yet [23]. Particularly, strict LMI-based condition has never been reported in the published works.

In this paper, by using a strict LMI approach, we study the robust stability and stabilization problems for a class of singular systems with a time-varying interval delay and uncertainties. Different from the existing results in $[13,19,21$, 23], first, the criteria proposed in our paper do not contain any semidefinite matrix inequality and are expressed as strict LMIs. Second, the new criteria are obtained by only using a well-known integral-inequality and do not employ any freeweighting matrix, which makes our methods more efficient. Third, a new type of uncertainty, namely, linear fractional form, is considered in this paper. Three numerical examples are given to illustrate the effectiveness of the presented method.

Notations. $\mathbf{R}^{n}$ denotes the $n$-dimensional Euclidean space, and $\mathbf{R}^{n \times m}$ denotes the sets of all $n \times m$ matrices. $I$ is the identity matrix with appropriate dimensions. For a real symmetric matrix $X, X^{\mathrm{T}}$ denotes its transpose, the notation $X \geq 0$ $(X>0)$ means that the matrix $X$ is positive semidefinite (positive-definite), and $\lambda_{\min }(X)\left(\lambda_{\max }(X)\right)$ denotes the minimum (maximum) eigenvalue of $X . C_{n, \tau}:=C\left([-\tau, 0], R^{n}\right)$ denotes the Banach space of continuous vector functions mapping the interval $[-\tau, 0]$ into $R^{n}, x_{t}:=x(t+\theta), \theta \in$ $[-\tau, 0]$, and $t \geq 0$ denotes the function family defined on $[-\tau, 0]$ which is generated by $n$-dimensional real vector valued continuous function $x(t), t \in[-\tau,+\infty)$. Obviously, $x_{t} \in$ $C_{n, \tau} \cdot\|\cdot\|$ refers to the Euclidean vector norm or spectral matrix norm, and $\|\varphi\|_{\mathcal{c}}:=\sup _{-\tau \leq t \leq 0}\|\varphi(t)\|$ stands for the norm of a function $\varphi \in C_{n, \tau}$. The symmetric terms in a symmetric matrix are denoted by $*$.

\section{Problem Formulation and Preliminaries}

Consider the following singular time-delay system:

$$
\begin{aligned}
E \dot{x}(t)= & (A+\Delta A) x(t)+\left(A_{\tau}+\Delta A_{\tau}\right) x(t-\tau(t)) \\
& +(B+\Delta B) u(t)
\end{aligned}
$$

$$
x(t)=\phi(t), \quad t \in\left[-h_{2}, 0\right]
$$

where $x(t) \in \mathbf{R}^{n}$ is the state vector, and $u(t) \in \mathbf{R}^{m}$ is the control input. $E, A \in \mathbf{R}^{n \times n}, A_{\tau} \in \mathbf{R}^{n \times n}$, and $B \in \mathbf{R}^{n \times m}$ are real constant matrices with appropriate dimensions, and $0<\operatorname{rank} E=r \leq n \cdot \tau(t)$ denotes the time-varying delay which satisfies $h_{1}<\tau(t) \leq h_{2}, \dot{\tau}(t) \leq \mu<1$. Note that $h_{1}$ may not be equal to $0 . \phi(t)$ is a compatible continuous vector-valued initial function on $\left[-h_{2}, 0\right] . \Delta A, \Delta A_{\tau}$ and $\Delta B$ are matrices with parametric uncertainties satisfying

$$
\begin{aligned}
& {\left[\begin{array}{lll}
\Delta A & \Delta A_{\tau} & \Delta B
\end{array}\right]=D \Delta(t)\left[\begin{array}{lll}
E_{a} & E_{\tau} & E_{b}
\end{array}\right],} \\
& \Delta(t)=F(t)(I-J F(t))^{-1}, \\
& I-J^{\mathrm{T}} J>0,
\end{aligned}
$$

where $D, E_{a}, E_{\tau}, E_{b}$, and $J$ are known real constant matrices of approximate dimensions and $F(t)$ are unknown time-varying matrix function satisfying

$$
F^{\mathrm{T}}(t) F(t) \leq I
$$

The parametric uncertainties $\Delta A, \Delta A_{\tau}$, and $\Delta B$ satisfying (2)-(5) are said to be admissible.

Remark 1. The above-structured linear fractional form includes the norm-bounded uncertainty as a special case when $J=0[3,8-11,13,19,23]$ and can describe a class of rational nonlinearities [32]. Note also that conditions (4) and (5) guarantee that $I-J F(t)$ is invertible.

The nominal unforced system of (2) can be written as

$$
\begin{gathered}
E \dot{x}(t)=A x(t)+A_{\tau} x(t-\tau(t)), \\
x(t)=\phi(t), \quad t \in\left[-h_{2}, 0\right] .
\end{gathered}
$$

The following notations are given.

(i) $S_{0}:=\left\{\phi(t) \in C_{n, h_{2}}, \phi(t)\right.$ is the compatible initial function of system $(6)\}$.

(ii) $S:=\left\{\phi(t) \in C_{n, h_{2}}\right.$, and there exists a uniquely continuous solution of system (6) on $[0,+\infty)$ for $\phi(t)\}$.

(iii) $B(0, \delta):=\left\{\phi(t) \mid \phi(t) \in C_{n, h_{2}},\|\phi\|_{c} \leq \delta, \delta>0\right\}$.

Definition 2 (see [33]). (1) The pair $(E, A)$ is said to be regular if $\operatorname{det}(s E-A)$ is not identically 0 .

(2) The pair $(E, A)$ is said to be impulse-free if $\operatorname{deg}(\operatorname{det}(s E-A))=\operatorname{rank} E$.

Lemma 3 (see [3]). If the pair $(E, A)$ is regular and impulse free, then for any compatible initial function $\phi(t) \in C_{n, h_{2}}$, there exists a uniquely continuous solution of system (6) on $[0,+\infty)$ for $\phi(t)$.

Definition 4 (see [3]). The singular system (6) is said to be regular and impulse free, if the pair $(E, A)$ is regular and impulse free. 
Definition 5 (see [11]). (1) The system (6) is said to be stable, if for any $\varepsilon>0$, there exists a scalar $\delta(\varepsilon)>0$ such that for any compatible initial function $\phi(t) \in B(0, \delta) \cap S$, the solution $x(t)$ of system (6) satisfies $\|x(t)\| \leq \varepsilon, t>0$.

(2) The system (6) is said to be asymptotically stable, if its zero solution is stable, and furthermore, there exists a $b_{0}>0$ such that for any compatible initial function $\phi(t) \in B\left(0, b_{0}\right) \cap$ $S$, the solution $x(t) \rightarrow 0$ as $t \rightarrow \infty$.

The objective of this note is to develop delay-rangedependent robust stability conditions for system (2) with $u(t)=0$ and to design a state-feedback controller

$$
u(t)=K x(t), \quad K \in \mathbf{R}^{m \times n}
$$

so that system (2) is closed-loop regular, impulse-free, and robustly asymptotically stable for admissible linear fractional form uncertainties. To this end, the following lemmas are needed.

Lemma 6 (see [34]). For any constant matrix $W \in \mathbf{R}^{n \times n}$, $W=W^{T}>0$, scalar $0 \leq r(t) \leq r_{M}$, and vector function $\dot{x}$ : $\left[-r_{M}, 0\right] \rightarrow \mathbf{R}^{n}$ such that the following integration is well defined, then

$$
\begin{aligned}
& -r_{M} \int_{-r(t)}^{0} \dot{x}^{T}(t+\xi) W \dot{x}(t+\xi) d \xi \\
& \quad \leq\left(x^{T}(t) x^{T}(t-r(t))\right)\left(\begin{array}{cc}
-W & W \\
W & -W
\end{array}\right)\left(\begin{array}{c}
x(t) \\
x(t-r(t))
\end{array}\right) .
\end{aligned}
$$

Lemma 7 (see [35]). Consider the function $\varphi: R^{+} \rightarrow R$, if $\dot{\varphi}$ is bounded on $[0, \infty)$; that is, there exists a scalar $\alpha>0$ such that $|\dot{\varphi}(t)| \leq \alpha$ for all $t \in[0, \infty)$, and then $\varphi$ is uniformly continuous on $[0, \infty)$.

Lemma 8 (see [35]). Consider the function $\varphi: R^{+} \rightarrow R$, if $\varphi(t)$ is uniformly continuous and $\int_{0}^{\infty} \varphi(s) d s<\infty$, then $\lim _{t \rightarrow \infty} \varphi(t)=0$.

Lemma 9 (see [32]). Given matrices $Q=Q^{T}, \Gamma$, and $\Xi$ of approximate dimensions, then

$$
Q+\Gamma \Delta(t) \Xi+\Xi^{T} \Delta^{T}(t) \Gamma^{T}<0,
$$

where $\Delta(t)$ is as in (3), if and only if there exists scalar $\varepsilon>0$ such that

$$
Q+\left[\begin{array}{ll}
\varepsilon^{-1} \Xi^{T} & \varepsilon \Gamma
\end{array}\right]\left[\begin{array}{cc}
I & -J \\
-J^{T} & I
\end{array}\right]^{-1}\left[\begin{array}{c}
\varepsilon^{-1} \Xi \\
\varepsilon \Gamma^{T}
\end{array}\right]<0 .
$$

\section{Stability Issue}

In this section, first of all, we will present new delay-rangedependent stability conditions that guarantee system (6) to be regular, impulse free, and asymptotically stable in terms of LMI, which will play a key role in obtaining the robust stability criterion for the uncertain system (2).
Theorem 10. Given scalars $0 \leq h_{1}<h_{2}$ and $\mu$, the singular system (6) is regular, impulse free, and asymptotically stable if there exist positive-definite matrices $P, Q_{i}, i=1,2,3, Z_{j}, j=$ 1,2 and matrix $S$ with appropriate dimensions such that

$$
\Lambda=\left[\begin{array}{cccccc}
\Lambda_{11} & \Lambda_{12} & 0 & 0 & h_{2} A^{T} Z_{1} & h_{12} A^{T} Z_{2} \\
* & \Lambda_{22} & \Lambda_{23} & \Lambda_{24} & h_{2} A_{\tau}^{T} Z_{1} & h_{12} A_{\tau}^{T} Z_{2} \\
* & * & \Lambda_{33} & 0 & 0 & 0 \\
* & * & * & \Lambda_{44} & 0 & 0 \\
* & * & * & * & -h_{2} Z_{1} & 0 \\
* & * & * & * & * & -h_{12} Z_{2}
\end{array}\right]<0
$$

where

$$
\begin{aligned}
& \Lambda_{11}= E^{T} P A+A^{T} P E+S R^{T} A+A^{T} R S^{T} \\
&+\sum_{i=1}^{3} Q_{i}-h_{2}^{-1} E^{T} Z_{1} E, \\
& \Lambda_{12}= E^{T} P A_{\tau}+S R^{T} A_{\tau}+h_{2}^{-1} E^{T} Z_{1} E, \\
& \Lambda_{22}=-(1-\mu) Q_{3}-h_{2}^{-1} E^{T} Z_{1} E \\
&-h_{12}^{-1} E^{T}\left(Z_{1}+Z_{2}\right) E-h_{12}^{-1} E^{T} Z_{2} E, \\
& \Lambda_{23}=h_{12}^{-1} E^{T} Z_{2} E, \\
& \Lambda_{24}=h_{12}^{-1} E^{T}\left(Z_{1}+Z_{2}\right) E, \\
& \Lambda_{33}=-Q_{1}-h_{12}^{-1} E^{T} Z_{2} E, \\
& \Lambda_{44}=-Q_{2}-h_{12}^{-1} E^{T}\left(Z_{1}+Z_{2}\right) E, \\
& h_{12}=h_{2}-h_{1},
\end{aligned}
$$

and $R \in \mathbf{R}^{n \times(n-r)}$ is any matrix with full column rank and satisfies $E^{T} R=0$.

Proof. Since rank $E=r \leq n$, there must exist two invertible matrices $\bar{G}$ and $\bar{H}$ such that

$$
\bar{E}=\bar{G} E \bar{H}=\left[\begin{array}{cc}
I_{r} & 0 \\
0 & 0
\end{array}\right] .
$$

Then, $R$ can be parameterized as

$$
R=\bar{G}^{\mathrm{T}}\left[\frac{0}{\Phi}\right]
$$

where $\bar{\Phi} \in \mathbf{R}^{(n-r) \times(n-r)}$ is any nonsingular matrix.

Similar to (13), we define

$$
\begin{gathered}
\bar{A}=\bar{G} A \bar{H}=\left[\begin{array}{ll}
\bar{A}_{11} & \bar{A}_{12} \\
\bar{A}_{21} & \bar{A}_{22}
\end{array}\right], \\
\bar{P}=\bar{G}^{-\mathrm{T}} P \bar{G}^{-1}=\left[\begin{array}{ll}
\bar{P}_{11} & \bar{P}_{12} \\
\bar{P}_{21} & \bar{P}_{22}
\end{array}\right], \\
\bar{S}=\bar{H}^{\mathrm{T}} S=\left[\begin{array}{l}
\bar{S}_{1} \\
\bar{S}_{2}
\end{array}\right], \quad \bar{R}=\bar{G}^{-\mathrm{T}} R=\left[\frac{0}{\Phi}\right], \\
\bar{Z}_{1}=\bar{G}^{-\mathrm{T}} Z_{1} \bar{G}^{-1}=\left[\begin{array}{ll}
\bar{Z}_{1,11} & \bar{Z}_{1,12} \\
\bar{Z}_{1,21} & \bar{Z}_{1,22}
\end{array}\right] .
\end{gathered}
$$


Since $\Lambda_{11}<0$ and $Q_{i}>0, i=1,2,3$, we can formulate the following inequality easily:

$$
\Psi=E^{\mathrm{T}} P A+A^{\mathrm{T}} P E+S R^{\mathrm{T}} A+A^{\mathrm{T}} R S^{\mathrm{T}}-h_{2}^{-1} E^{\mathrm{T}} Z_{1} E<0 .
$$

Pre- and postmultiplying $\Psi<0$ by $\bar{H}^{\mathrm{T}}$ and $\bar{H}$, respectively, yields

$$
\bar{\Psi}=\bar{H}^{\mathrm{T}} \Psi \bar{H}=\left[\begin{array}{cc}
\bar{\Psi}_{11} & \bar{\Psi}_{12} \\
* & \bar{A}_{22}^{\mathrm{T}} \bar{\Phi} \bar{S}_{2}^{\mathrm{T}}+\bar{S}_{2} \bar{\Phi}^{\mathrm{T}} \bar{A}_{22}
\end{array}\right]<0,
$$

where $\bar{\Psi}_{11}$ and $\bar{\Psi}_{12}$ represent the matrices not relevant in the following discussion. From (17), it is easy to see that

$$
\bar{A}_{22}^{\mathrm{T}} \bar{\Phi} \bar{S}_{2}^{\mathrm{T}}+\bar{S}_{2} \bar{\Phi}^{\mathrm{T}} \bar{A}_{22}<0
$$

which gives that $\bar{A}_{22}$ is nonsingular.

Define

$$
\widetilde{G}=\left[\begin{array}{cc}
I & -\bar{A}_{12} \bar{A}_{22}^{-1} \\
0 & I
\end{array}\right] \bar{G}, \quad \widetilde{H}=\bar{H}\left[\begin{array}{cc}
I & 0 \\
-\bar{A}_{22}^{-1} \bar{A}_{21} & \bar{A}_{22}^{-1}
\end{array}\right] .
$$

After some algebraic manipulations, we can obtain

$$
\widetilde{E}=\widetilde{G} E \widetilde{H}=\left[\begin{array}{cc}
I_{r} & 0 \\
0 & 0
\end{array}\right], \quad \widetilde{A}=\widetilde{G} A \widetilde{H}=\left[\begin{array}{cc}
\widetilde{A}_{11} & 0 \\
0 & I
\end{array}\right],
$$

where $\widetilde{A}_{11}=\bar{A}_{11}-\bar{A}_{12} \bar{A}_{22}^{-1} \bar{A}_{21}$. Then, it can be shown that

$$
\begin{aligned}
\operatorname{det}(s E-A) & =\operatorname{det}\left(\widetilde{G}^{-1}\right) \operatorname{det}(s \widetilde{E}-\widetilde{A}) \operatorname{det}\left(\widetilde{H}^{-1}\right) \\
& =\operatorname{det}\left(\widetilde{G}^{-1}\right)(-1)^{(n-r)} \operatorname{det}\left(s I_{r}-\widetilde{A}_{11}\right) \operatorname{det}\left(\widetilde{H}^{-1}\right)
\end{aligned}
$$

which implies that $\operatorname{det}(s E-A)$ is not identically zero and $\operatorname{deg}(\operatorname{det}(s E-A))=r=\operatorname{rank} E$. Then, the pair of $(E, A)$ is regular and impulse-free, which shows that system (6) is regular and impulse-free. In the following, we will prove that system (6) is also asymptotically stable.

Denote

$$
\begin{gathered}
\widetilde{A}_{\tau}=\widetilde{G} A_{\tau} \widetilde{H}=\left[\begin{array}{ll}
\widetilde{A}_{\tau 11} & \widetilde{A}_{\tau 12} \\
\widetilde{A}_{\tau 21} & \widetilde{A}_{\tau 22}
\end{array}\right], \\
\widetilde{P}=\widetilde{G}^{-\mathrm{T}} P \widetilde{G}^{-1}=\left[\begin{array}{ll}
\widetilde{P}_{11} & \widetilde{P}_{12} \\
\widetilde{P}_{21} & \widetilde{P}_{22}
\end{array}\right], \\
\widetilde{R}=\widetilde{G}^{-\mathrm{T}} R=\left[\begin{array}{c}
0 \\
\widetilde{\Phi}
\end{array}\right], \quad \quad \quad \widetilde{S}^{2} \widetilde{H}^{\mathrm{T}} S=\left[\begin{array}{c}
\widetilde{S}_{1} \\
\widetilde{S}_{2}
\end{array}\right], \\
\widetilde{Q}_{i}=\widetilde{H}^{\mathrm{T}} Q_{i} \widetilde{H}=\left[\begin{array}{ll}
\widetilde{Q}_{i, 11} & \widetilde{Q}_{i, 12} \\
\widetilde{Q}_{i, 21} & \widetilde{Q}_{i, 22}
\end{array}\right], \quad i=1,2,3, \\
\widetilde{Z}_{j}=\widetilde{G}^{-\mathrm{T}} Z_{j} \widetilde{G}^{-1}=\left[\begin{array}{ll}
\widetilde{Z}_{j, 11} & \widetilde{Z}_{j, 12} \\
\widetilde{Z}_{j, 21} & \widetilde{Z}_{j, 22}
\end{array}\right], \quad j=1,2 .
\end{gathered}
$$

By using Schur complement and noting that $Q_{i}>0, i=$ $1,2, \mu<1$, it follows from (11) that

$$
\left[\begin{array}{cc}
\bar{\Lambda}_{11} & \Lambda_{12} \\
* & \bar{\Lambda}_{22}
\end{array}\right]<0
$$

where

$$
\begin{aligned}
& \bar{\Lambda}_{11}=E^{\mathrm{T}} P A+A^{\mathrm{T}} P E+S R^{\mathrm{T}} A+A^{\mathrm{T}} R S^{\mathrm{T}}+Q_{3}-h_{2}^{-1} E^{\mathrm{T}} Z_{1} E, \\
& \bar{\Lambda}_{22}=-Q_{3}-h_{2}^{-1} E^{\mathrm{T}} Z_{1} E-h_{12}^{-1} E^{\mathrm{T}}\left(Z_{1}+Z_{2}\right) E-h_{12}^{-1} E^{\mathrm{T}} Z_{2} E .
\end{aligned}
$$

Pre- and post-multiplying (23) by $\operatorname{diag}\left\{\widetilde{H}^{\mathrm{T}}, \widetilde{H}^{\mathrm{T}}\right\}$ and $\operatorname{diag}\{\widetilde{H}$, $\widetilde{H}\}$, respectively, yields

$$
\left[\begin{array}{cc}
\widetilde{\bar{\Lambda}}_{11} & \widetilde{\Lambda}_{12} \\
* & \widetilde{\bar{\Lambda}}_{22}
\end{array}\right]<0
$$

where

$\widetilde{\bar{\Lambda}}_{11}=\widetilde{E}^{\mathrm{T}} \widetilde{P} \widetilde{A}+\widetilde{A}^{\mathrm{T}} \widetilde{P} \widetilde{E}+\widetilde{S} \widetilde{R}^{\mathrm{T}} \widetilde{A}+\widetilde{A}^{\mathrm{T}} \widetilde{R} \widetilde{S}^{\mathrm{T}}+\widetilde{Q}_{3}-h_{2}^{-1} \widetilde{E}^{\mathrm{T}} \widetilde{Z} \widetilde{E}$,

$\widetilde{\Lambda}_{12}=\widetilde{E}^{\mathrm{T}} \widetilde{P} \widetilde{A}_{\tau}+\widetilde{S} \widetilde{R}^{\mathrm{T}} \widetilde{A}_{\tau}+h_{2}^{-1} \widetilde{E}^{\mathrm{T}} \widetilde{Z}_{1} \widetilde{E}$

$\widetilde{\bar{\Lambda}}_{22}=-\widetilde{Q}_{3}-h_{2}^{-1} \widetilde{E}^{\mathrm{T}} \widetilde{Z}_{1} \widetilde{E}-h_{12}^{-1} \widetilde{E}^{\mathrm{T}}\left(\widetilde{Z}_{1}+\widetilde{Z}_{2}\right) \widetilde{E}-h_{12}^{-1} \widetilde{E}^{\mathrm{T}} \widetilde{Z}_{2} \widetilde{E}$.

Substituting (20), (22) into (25), we have

$$
\left[\begin{array}{cc}
\widetilde{S}_{2} \widetilde{\Phi}^{\mathrm{T}}+\widetilde{\Phi}^{\widetilde{S}_{2}^{\mathrm{T}}+\widetilde{Q}_{3,22}} & \widetilde{S}_{2} \widetilde{\Phi}^{\mathrm{T}} \widetilde{A}_{\tau 22}^{\mathrm{T}} \\
* & -\widetilde{Q}_{3,22}
\end{array}\right]<0 .
$$

Pre- and post-multiplying (27) by $\xi$ = $\left[\widetilde{\Phi}^{-\mathrm{T}} \widetilde{S}_{2}^{-1}-\widetilde{A}_{\tau 22} \widetilde{\Phi}^{-\mathrm{T}} \widetilde{S}_{2}^{-1}\right]$ and $\xi^{\mathrm{T}}$, respectively, yields

$$
\widetilde{A}_{\tau 22} \widetilde{\Theta} \widetilde{A}_{\tau 22}^{\mathrm{T}}-\widetilde{\Theta}<0,
$$

where

$$
\widetilde{\Theta}=-\widetilde{\Phi}^{-\mathrm{T}} \widetilde{S}_{2}^{-1}\left(\widetilde{S}_{2} \widetilde{\Phi}^{\mathrm{T}}+\widetilde{\Phi} \widetilde{S}_{2}^{\mathrm{T}}+\widetilde{Q}_{3,22}\right) \widetilde{S}_{2}^{-\mathrm{T}} \widetilde{\Phi}^{-1}>0 .
$$

Therefore,

$$
\rho\left(\widetilde{A}_{\tau 22}\right)<1
$$

Now, let

$$
\tilde{x}(t)=\left[\begin{array}{l}
\tilde{x}_{1}(t) \\
\tilde{x}_{2}(t)
\end{array}\right]=\bar{H}^{-1} x(t)
$$

where $\tilde{x}_{1}(t) \in \mathbf{R}^{r}$ and $\tilde{x}_{2}(t) \in \mathbf{R}^{n-r}$. Using the expressions in (20), (22), and (31), system (6) can be decomposed as

$$
\begin{gathered}
\dot{\tilde{x}}_{1}(t)=\widetilde{A}_{11} \tilde{x}_{1}(t)+\widetilde{A}_{\tau 11} \tilde{x}_{1}(t-\tau(t))+\widetilde{A}_{\tau 12} \tilde{x}_{2}(t-\tau(t)), \\
0=\tilde{x}_{2}(t)+\widetilde{A}_{\tau 21} \tilde{x}_{1}(t-\tau(t))+\widetilde{A}_{\tau 22} \tilde{x}_{2}(t-\tau(t)),
\end{gathered}
$$


or equivalently rewritten as

$$
\widetilde{E} \dot{\tilde{x}}(t)=\widetilde{A} \widetilde{x}(t)+\widetilde{A}_{\tau} \tilde{x}(t-\tau(t)) .
$$

It is easy to see that the stability of system (6) is equivalent to that of system (34).

Construct the Lyapunov-Krasovskii functional for system (34) as

$$
\begin{aligned}
\widetilde{V}\left(\widetilde{x}_{t}\right)= & \widetilde{x}^{\mathrm{T}}(t) \widetilde{E}^{\mathrm{T}} \widetilde{P} \widetilde{E} \widetilde{x}(t) \\
& +\sum_{i=1}^{2} \int_{t-h_{i}}^{t} \widetilde{x}^{\mathrm{T}}(s) \widetilde{\mathrm{Q}}_{i} \widetilde{x}(s) d s \\
& +\int_{t-\tau(t)}^{t} \widetilde{x}^{\mathrm{T}}(s) \widetilde{\mathrm{Q}}_{3} \widetilde{x}(s) d s \\
& +\int_{-h_{2}}^{0} \int_{t+\theta}^{t} \dot{\tilde{x}}^{\mathrm{T}}(s) \widetilde{E}^{\mathrm{T}} \widetilde{Z}_{1} \widetilde{E} \dot{\tilde{x}}(s) d s d \theta \\
& +\int_{-h_{2}}^{-h_{1}} \int_{t+\theta}^{t} \dot{\bar{x}}^{\mathrm{T}}(s) \widetilde{E}^{\mathrm{T}} \widetilde{Z}_{2} \widetilde{E} \dot{\bar{x}}(s) d s d \theta .
\end{aligned}
$$

By Lemma 6, the following inequalities are true

$$
\begin{aligned}
& -h_{2} \int_{t-h_{2}}^{t} \dot{\tilde{x}}^{\mathrm{T}}(s) \widetilde{E}^{\mathrm{T}} \widetilde{Z}_{1} \widetilde{E} \dot{\tilde{x}}(s) d s \\
& =-h_{2} \int_{t-\tau(t)}^{t} \dot{\tilde{x}}^{\mathrm{T}}(s) \widetilde{E}^{\mathrm{T}} \widetilde{Z}_{1} \widetilde{E} \dot{\tilde{x}}(s) d s \\
& -h_{2} \int_{t-h_{2}}^{t-\tau(t)} \dot{\tilde{x}}^{\mathrm{T}}(s) \widetilde{E}^{\mathrm{T}} \widetilde{Z}_{1} \widetilde{E} \dot{\tilde{x}}(s) d s \\
& \leq\left(\tilde{x}^{\mathrm{T}}(t) \tilde{x}^{\mathrm{T}}(t-\tau(t))\right) \\
& \times\left(\begin{array}{cc}
-\widetilde{E}^{\mathrm{T}} \widetilde{Z}_{1} \widetilde{E} & \widetilde{E}^{\mathrm{T}} \widetilde{Z}_{1} \widetilde{E} \\
\widetilde{E}^{\mathrm{T}} \widetilde{Z}_{1} \widetilde{E} & -\widetilde{E}^{\mathrm{T}} \widetilde{Z}_{1} \widetilde{E}
\end{array}\right)\left(\begin{array}{c}
\tilde{x}(t) \\
\tilde{x}(t-\tau(t))
\end{array}\right) \\
& +h_{12}^{-1}\left(\tilde{x}^{\mathrm{T}}(t-\tau(t)) \tilde{x}^{\mathrm{T}}\left(t-h_{2}\right)\right) \\
& \times\left(\begin{array}{cc}
-\widetilde{E}^{\mathrm{T}} \widetilde{Z}_{1} \widetilde{E} & \widetilde{E}^{\mathrm{T}} \widetilde{Z}_{1} \widetilde{E} \\
\widetilde{E}^{\mathrm{T}} \widetilde{Z}_{1} \widetilde{E} & -\widetilde{E}^{\mathrm{T}} \widetilde{Z}_{1} \widetilde{E}
\end{array}\right)\left(\begin{array}{c}
\tilde{x}(t-\tau(t)) \\
\widetilde{x}\left(t-h_{2}\right)
\end{array}\right), \\
& -h_{12} \int_{t-h_{2}}^{t-h_{1}} \dot{\tilde{x}}^{\mathrm{T}}(s) \widetilde{E}^{\mathrm{T}} \widetilde{Z}_{2} \widetilde{E} \dot{\tilde{x}}(s) d s \\
& =-h_{12} \int_{t-h_{2}}^{t-\tau(t)} \dot{\tilde{x}}^{\mathrm{T}}(s) \widetilde{E}^{\mathrm{T}} \widetilde{Z}_{2} \widetilde{E} \dot{\tilde{x}}(s) d s \\
& -h_{12} \int_{t-\tau(t)}^{t-h_{1}} \dot{\tilde{x}}^{\mathrm{T}}(s) \widetilde{E}^{\mathrm{T}} \widetilde{Z}_{2} \widetilde{E} \dot{\tilde{x}}(s) d s
\end{aligned}
$$

$$
\begin{aligned}
& \leq\left(\tilde{x}^{\mathrm{T}}(t-\tau(t)) \tilde{x}^{\mathrm{T}}\left(t-h_{2}\right)\right) \\
& \times\left(\begin{array}{cc}
-\widetilde{E}^{\mathrm{T}} \widetilde{Z}_{2} \widetilde{E} & \widetilde{E}^{\mathrm{T}} \widetilde{Z}_{2} \widetilde{E} \\
\widetilde{E}^{\mathrm{T}} \widetilde{Z}_{2} \widetilde{E} & -\widetilde{E}^{\mathrm{T}} \widetilde{Z}_{2} \widetilde{E}
\end{array}\right)\left(\begin{array}{c}
\tilde{x}(t-\tau(t)) \\
\tilde{x}\left(t-h_{2}\right)
\end{array}\right) \\
& +\left(\tilde{x}^{\mathrm{T}}\left(t-h_{1}\right) \tilde{x}^{\mathrm{T}}(t-\tau(t))\right) \\
& \times\left(\begin{array}{cc}
-\widetilde{E}^{\mathrm{T}} \widetilde{Z}_{2} \widetilde{E} & \widetilde{E}^{\mathrm{T}} \widetilde{Z}_{2} \widetilde{E} \\
\widetilde{E}^{\mathrm{T}} \widetilde{Z}_{2} \widetilde{E} & -\widetilde{E}^{\mathrm{T}} \widetilde{Z}_{2} \widetilde{E}
\end{array}\right)\left(\begin{array}{c}
\tilde{x}\left(t-h_{1}\right) \\
\widetilde{x}(t-\tau(t))
\end{array}\right) .
\end{aligned}
$$

On the other hand, noticing that $E^{\mathrm{T}} R=0$, we can deduce that

$$
0=2 \tilde{x}^{\mathrm{T}}(t) \widetilde{S} \widetilde{R}^{\mathrm{T}} \widetilde{E} \dot{\tilde{x}}(t),
$$

where $\widetilde{S}$ is any matrix with appropriate dimensions.

Taking the derivative of $\widetilde{V}\left(\widetilde{x}_{t}\right)$ with respect to $t$ along the trajectory of system (34) and using (36) and (37), we have

$\dot{\widetilde{V}}\left(\tilde{x}_{t}\right)$

$$
\begin{aligned}
= & \dot{\tilde{x}}^{\mathrm{T}}(t) \widetilde{E}^{\mathrm{T}} \widetilde{P} \widetilde{E} \widetilde{x}(t)+\widetilde{x}^{\mathrm{T}}(t) \widetilde{E}^{\mathrm{T}} \widetilde{P} \widetilde{E} \dot{\tilde{x}}(t) \\
& +\sum_{i=1}^{2}\left\{\widetilde{x}^{\mathrm{T}}(t) \widetilde{Q_{i}} \widetilde{x}(t)-\widetilde{x}^{\mathrm{T}}\left(t-h_{i}\right) \widetilde{Q}_{i} \widetilde{x}\left(t-h_{i}\right)\right\} \\
& +\widetilde{x}^{\mathrm{T}}(t) \widetilde{Q}_{3} \widetilde{x}(t)-(1-\dot{\tau}(t)) \widetilde{x}^{\mathrm{T}}(t-\tau(t)) \widetilde{Q}_{3} \widetilde{x}(t-\tau(t)) \\
& +h_{2} \dot{\tilde{x}}^{\mathrm{T}}(t) \widetilde{E}^{\mathrm{T}} \widetilde{Z}_{1} \widetilde{E} \dot{\bar{x}}(t)-\int_{t-h_{2}}^{t} \dot{\tilde{x}}^{\mathrm{T}}(s) \widetilde{E}^{\mathrm{T}} \widetilde{Z}_{1} \widetilde{E} \dot{\tilde{x}}(s) d s \\
& +\left(h_{2}-h_{1}\right) \dot{\tilde{x}}^{\mathrm{T}}(t) \widetilde{E}^{\mathrm{T}} \widetilde{Z}_{2} \widetilde{E} \dot{\tilde{x}}(t)-\int_{t-h_{2}}^{t-h_{1}} \dot{\tilde{x}}^{\mathrm{T}}(s) \widetilde{E}^{\mathrm{T}} \widetilde{Z_{2}} \widetilde{E} \dot{\tilde{x}}(s) d s \\
\leq & \widetilde{\psi}^{\mathrm{T}}(t) \widetilde{\Lambda} \widetilde{\psi}(t),
\end{aligned}
$$

where

$$
\begin{gathered}
\tilde{\psi}(t)=\left[\begin{array}{llll}
\tilde{x}^{\mathrm{T}}(t) & \tilde{x}^{\mathrm{T}}(t-\tau(t)) & \tilde{x}^{\mathrm{T}}\left(t-h_{1}\right) & \tilde{x}^{\mathrm{T}}\left(t-h_{2}\right)
\end{array}\right]^{\mathrm{T}}, \\
\widetilde{\Lambda}=\left[\begin{array}{cccc}
\widetilde{\Lambda}_{11} & \widetilde{\Lambda}_{12} & 0 & 0 \\
* & \widetilde{\Lambda}_{22} & \widetilde{\Lambda}_{23} & \widetilde{\Lambda}_{24} \\
* & * & \widetilde{\Lambda}_{33} & 0 \\
* & * & * & \widetilde{\Lambda}_{44}
\end{array}\right],
\end{gathered}
$$

with

$$
\begin{aligned}
\widetilde{\Lambda}_{11}= & \widetilde{E}^{\mathrm{T}} \widetilde{P} \widetilde{A}+\widetilde{A}^{\mathrm{T}} \widetilde{P} \widetilde{E}+\widetilde{S} \widetilde{R}^{\mathrm{T}} \widetilde{A}+\widetilde{A}^{\mathrm{T}} \widetilde{R} \widetilde{S}^{\mathrm{T}} \\
& +\sum_{i=1}^{3} \widetilde{Q}_{i}+h_{2} \widetilde{A}^{\mathrm{T}} \widetilde{Z}_{1} \widetilde{A}+h_{12} \widetilde{A}^{\mathrm{T}} \widetilde{Z}_{2} \widetilde{A}-h_{2}^{-1} \widetilde{E}^{\mathrm{T}} \widetilde{Z}_{1} \widetilde{E} \\
\widetilde{\Lambda}_{12}= & \widetilde{E}^{\mathrm{T}} \widetilde{P} \widetilde{A}_{\tau}+\widetilde{S} \widetilde{R}^{\mathrm{T}} \widetilde{A}_{\tau}+h_{2} \widetilde{A}^{\mathrm{T}} \widetilde{Z}_{1} \widetilde{A}_{\tau}+h_{12} \widetilde{A}^{\mathrm{T}} \widetilde{Z}_{2} \widetilde{A}_{\tau} \\
& +h_{2}^{-1} \widetilde{E}^{\mathrm{T}} \widetilde{Z}_{1} \widetilde{E}, \\
\widetilde{\Lambda}_{22}= & -(1-\mu) \widetilde{Q}_{3}+h_{2} \widetilde{A}_{\tau}^{\mathrm{T}} \widetilde{Z}_{1} \widetilde{A}_{\tau}+h_{12} \widetilde{A}_{\tau}^{\mathrm{T}} \widetilde{Z}_{2} \widetilde{A}_{\tau} \\
& -h_{2}^{-1} \widetilde{E}^{\mathrm{T}} \widetilde{Z}_{1} \widetilde{E}-h_{12}^{-1} \widetilde{E}^{\mathrm{T}}\left(\widetilde{Z}_{1}+\widetilde{Z}_{2}\right) \widetilde{E}-h_{12}^{-1} \widetilde{E}^{\mathrm{T}} \widetilde{Z_{2}} \widetilde{E},
\end{aligned}
$$




$$
\begin{gathered}
\widetilde{\Lambda}_{23}=h_{12}^{-1} \widetilde{E}^{\mathrm{T}} \widetilde{Z}_{2} \widetilde{E}, \quad \widetilde{\Lambda}_{24}=h_{12}^{-1} \widetilde{E}^{\mathrm{T}}\left(\widetilde{Z}_{1}+\widetilde{Z}_{2}\right) \widetilde{E} \\
\widetilde{\Lambda}_{33}=-\widetilde{Q}_{1}-h_{12}^{-1} \widetilde{E}^{\mathrm{T}} \widetilde{Z}_{2} \widetilde{E} \\
\widetilde{\Lambda}_{44}=-\widetilde{Q}_{2}-h_{12}^{-1} \widetilde{E}^{\mathrm{T}}\left(\widetilde{Z}_{1}+\widetilde{Z}_{2}\right) \widetilde{E}
\end{gathered}
$$

It is easy to see that (17) guarantees $\dot{\widetilde{V}}\left(\widetilde{x}_{t}\right)<0$ and

$$
\begin{aligned}
\lambda_{1}\left\|\tilde{x}_{1}(t)\right\|^{2}-\widetilde{V}(\tilde{x}(0)) & \leq-\widetilde{V}(\tilde{x}(t))-\widetilde{V}(\tilde{x}(0)) \\
& =\int_{0}^{t} \dot{\tilde{V}}(\tilde{x}(s)) d s \leq-\lambda_{2} \int_{0}^{t}\|\widetilde{x}(s)\|^{2} d s \\
& \leq-\lambda_{2} \int_{0}^{t}\left\|\widetilde{x}_{1}(s)\right\|^{2} d s<0,
\end{aligned}
$$

where $\lambda_{1}=\lambda_{\min }\left(\widetilde{P}_{11}\right)>0, \lambda_{1}=-\lambda_{\max }(\widetilde{\Lambda})>0$.

Taking into account (41), we can deduce that

$$
\lambda_{1}\left\|\widetilde{x}_{1}(t)\right\|^{2}+\lambda_{2} \int_{0}^{t}\left\|\widetilde{x}_{1}(s)\right\|^{2} d s \leq \widetilde{V}(\tilde{x}(0)) .
$$

Therefore,

$$
\begin{gathered}
\left\|\tilde{x}_{1}(t)\right\|^{2} \leq m_{1}, \\
\int_{0}^{t}\left\|\tilde{x}_{1}(s)\right\|^{2} d s \leq m_{2},
\end{gathered}
$$

where $m_{1}=\left(1 / \lambda_{1}\right) \widetilde{V}(\widetilde{x}(0))>0, m_{2}=\left(1 / \lambda_{2}\right) \widetilde{V}(\tilde{x}(0))>$ 0 . Thus, $\left\|\tilde{x}_{1}(t)\right\|$ is bounded. Considering this and (30), it can be deduced from (33) that $\left\|\widetilde{x}_{2}(t)\right\|$ is bounded; hence, it follows that from (32) that $\left\|\dot{\tilde{x}}_{1}(t)\right\|$ is bounded. Therefore, $(d / d t)\left\|\dot{\tilde{x}}_{1}(t)\right\|^{2}$ is bounded too. By Lemma 7, we obtain that $\left\|\dot{\tilde{x}}_{1}(t)\right\|^{2}$ is uniformly continuous. Therefore, noting (44) and using Lemma 8, we get

$$
\lim _{t \rightarrow \infty}\left\|\tilde{x}_{1}(t)\right\|=0 .
$$

This, together with (33), implies that

$$
\lim _{t \rightarrow \infty}\left\|\tilde{x}_{2}(t)\right\|=0 \text {. }
$$

Thus, according to Definition 5, system (34) is stable. This completes the proof.

Remark 11. From the proof of Theorem 10, it is clear to see that neither model transformation nor bounding technique for cross-terms is involved. Hence, the conservatism inherited from these ideas will no longer exist in Theorem 10.

Remark 12. Free-weighting matrices in [11-13, 22, 23, 25] plays an important role to reducing the conservatism of delaydependent stability conditions. However, too many freeweighting matrices will complicate the system analysis and increase the computational demand. It is worth pointing out that no free-weighting matrix is involved in Theorem 10.
Remark 13. Recently, the delay-partitioning or delay-fractioning method [36] was widely used to reduce the conservatism of the delay-dependent results of standard time-delay systems. This method can be extended to study the stability of singular time-delay system (2). Suppose we decompose the delay interval $\left[h_{1}, h_{2}\right]$ into Nequidistant subintervals. Defining $h^{i}=h_{1}+i\left(h_{2}-h_{1}\right) / N, i=1,2, \ldots, N-1$, and constructing the following Lyapunov-Krasovskii functional:

$$
\begin{aligned}
\widetilde{V}\left(\widetilde{x}_{t}\right)= & \tilde{x}^{\mathrm{T}}(t) \widetilde{E}^{\mathrm{T}} \widetilde{P} \widetilde{E} \tilde{x}(t)+\sum_{i=1}^{2} \int_{t-h_{i}}^{t} \tilde{x}^{\mathrm{T}}(s) \widetilde{Q}_{i} \widetilde{x}(s) d s \\
& +\sum_{j=1}^{N-1} \int_{t-h^{j}}^{t} x^{\mathrm{T}}(s) \widetilde{Q}_{j}^{\prime} x(s) d s \\
& +\int_{-h_{2}}^{0} \int_{t+\theta}^{t} \dot{\tilde{x}}^{\mathrm{T}}(s) \widetilde{E}^{\mathrm{T}} \widetilde{Z}_{1} \widetilde{E} \dot{\tilde{x}}(s) d s d \theta \\
& +\int_{-h^{1}}^{-h_{1}} \int_{t+\theta}^{t} \dot{\tilde{x}}^{\mathrm{T}}(s) \widetilde{E}^{\mathrm{T}} \widetilde{Z}_{2} \widetilde{E} \dot{\tilde{x}}(s) d s d \theta \\
& +\sum_{l=1}^{N-2} \int_{-h^{l+1}}^{-h^{l}} \int_{t+\theta}^{t} \dot{\dot{x}}^{\mathrm{T}}(s) \widetilde{E}^{\mathrm{T}} \widetilde{Z}_{l+2} \widetilde{E} \dot{\tilde{x}}(s) d s d \theta \\
& +\int_{-h_{2}}^{-h^{N-1}} \int_{t+\theta}^{t} \dot{\tilde{x}}^{\mathrm{T}}(s) \widetilde{E}^{\mathrm{T}} \widetilde{Z}_{N+1} \widetilde{E} \dot{\tilde{x}}(s) d s d \theta
\end{aligned}
$$

with $\widetilde{Q}_{1}>0, \widetilde{Q}_{2}>0, \widetilde{Q}_{j}^{\prime}>0, j=1,2, \ldots, N-1, \widetilde{Z}_{1}>0$, $\widetilde{Z}_{2}>0$ and $\widetilde{Z}_{l+2}>0, l=1,2, \ldots, N-2$. Then, by checking the variation of $\widetilde{V}\left(\widetilde{x}_{t}\right)$ for the case when $\tau(t) \in\left[h_{1}, h^{1}\right]$ or $\tau(t) \in\left[h^{j}, h^{j+1}\right](j=1,2, \ldots, N-1)$ or $\tau(t) \in\left[h^{N-1}, h_{2}\right]$, respectively, we can derive the delay-dependent condition, which can guarantee that $\widetilde{V}\left(\widetilde{x}_{t}\right)<0$. Generally, increasing $N$ may result in the reduction of conservatism of the obtained results. However, the corresponding computational complexity will be increased greatly since the dimensions and matrix variables of the involved LMIs will be sharply expanded. For example, in [36], a numerical example has shown that, with $N$ changing from 1 to 3 , the allowable upper bounds of $h_{2}$ increased $12.9 \%$, but the consumed CPU time increased 9 times.

Theorem 10 presents a delay-range-dependent criterion for system (6) with time-varying delay $\tau(t)$ in a range. If we set $Q_{1}=0$ and $Z_{2}=0$, Theorem 10 yields the following delaydependent stability criterion.

Corollary 14. Given scalars $h_{2}>0, h_{1}=0$ and $\mu$, system (6) is regular, impulse free, and asymptotically stable if there exist positive-definite matrices $P, Q_{i}, i=2,3, Z_{1}$, and matrix $S$ with appropriate dimensions such that

$$
\left[\begin{array}{cccc}
T_{11} & T_{12} & 0 & h_{2} A^{T} Z_{1} \\
* & T_{22} & h_{2}^{-1} E^{T} Z_{1} E & h_{2} A_{\tau}^{T} Z_{1} \\
* & * & T_{33} & 0 \\
* & * & * & -h_{2} Z_{1}
\end{array}\right]
$$


where

$$
\begin{aligned}
\mathrm{T}_{11}= & E^{T} P A+A^{T} P E+S R^{T} A+A^{T} R S^{T} \\
& +\sum_{i=2}^{3} Q_{i}-h_{2}^{-1} E^{T} Z_{1} E \\
\mathrm{~T}_{12}= & E^{T} P A_{\tau}+S R^{T} A_{\tau}+h_{2}^{-1} E^{T} Z_{1} E, \\
\mathrm{~T}_{22}= & -(1-\mu) Q_{3}-2 h_{2}^{-1} E^{T} Z_{1} E, \\
\mathrm{~T}_{33}= & -Q_{2}-h_{2}^{-1} E^{T} Z_{1} E,
\end{aligned}
$$

and $R \in \mathbf{R}^{n \times(n-r)}$ is any matrix with full column rank and satisfies $E^{T} R=0$.

Now, we will present the delay-range-dependent robust stability conditions for the uncertain singular time-delay system (2) with $u(t)=0$ via Theorem 10 .

Theorem 15. Given scalars $0 \leq h_{1}<h_{2}$ and $\mu$, the uncertain singular time-delay system (2) with $u(t)=0$ is regular, impulse free, and robustly asymptotically stable if there exist positivedefinite matrices $P, Q_{i}, i=1,2,3, Z_{j}, j=1,2$, and matrix $S$ with appropriate dimensions and a scalar $\eta>0$ such that the following LMI holds:

$$
\begin{gathered}
{\left[\begin{array}{cccccccc}
\Lambda_{11} & \Lambda_{12} & 0 & 0 & h_{2} A^{T} Z_{1} & h_{12} A^{T} Z_{2} & \eta E_{a}^{T} & \left(E^{T} P+S R^{T}\right) D \\
* & \Lambda_{22} & \Lambda_{23} & \Lambda_{24} & h_{2} A_{\tau}^{T} Z_{1} & h_{12} A_{\tau}^{T} Z_{2} & \eta E_{\tau}^{T} & 0 \\
* & * & \Lambda_{33} & 0 & 0 & 0 & 0 & 0 \\
* & * & * & \Lambda_{44} & 0 & 0 & 0 & 0 \\
* & * & * & * & -h_{2} Z_{1} & 0 & 0 & h_{2} Z_{1} D \\
* & * & * & * & * & -h_{12} Z_{2} & 0 & h_{12} Z_{2} D \\
* & * & * & * & * & * & -\eta I & \eta J \\
* & * & * & * & * & * & * & -\eta I
\end{array}\right]} \\
\\
\quad<0,
\end{gathered}
$$

where $\Lambda_{11}, \Lambda_{12}, \Lambda_{22}, \Lambda_{23}, \Lambda_{24}, \Lambda_{33}, \Lambda_{44}$, and $h_{12}$ are defined in (11).

Proof. Suppose (50) to be true. Let $\varepsilon=1 / \sqrt{\eta}$. Pre- and postmultiplying the left-hand side matrix of (50) by $\operatorname{diag}\{I, I$, $I, I, I, I, \mathcal{\varepsilon}, \varepsilon\}$ and its transpose, respectively, we obtain

$$
\Lambda+\left[\begin{array}{ll}
\varepsilon^{-1} \Xi^{\mathrm{T}} & \varepsilon \Gamma
\end{array}\right]\left[\begin{array}{cc}
I & -J \\
-J^{\mathrm{T}} & I
\end{array}\right]^{-1}\left[\begin{array}{c}
\varepsilon^{-1} \Xi \\
\varepsilon \Gamma^{\mathrm{T}}
\end{array}\right]<0,
$$

where

$$
\begin{aligned}
& \Gamma=\left[\begin{array}{llllll}
D^{\mathrm{T}}\left(P E+R S^{\mathrm{T}}\right) & 0 & 0 & 0 & h_{2} D^{\mathrm{T}} Z_{1} & h_{12} D^{\mathrm{T}} Z_{2}
\end{array}\right]^{\mathrm{T}}, \\
& \Xi=\left[\begin{array}{llllll}
E_{a} & E_{\tau} & 0 & 0 & 0 & 0
\end{array}\right],
\end{aligned}
$$

and $\Lambda$ is defined in (11). Thus, $\Theta=\Lambda+\Gamma \Delta(t) \Xi+\Xi^{\mathrm{T}} \Delta^{\mathrm{T}}(t) \Gamma^{\mathrm{T}}<$ 0 holds according to Lemma 9. It can be verified that $\Theta$ is exactly the left-hand side of (11) when $A$ and $A_{\tau}$ are replaced with $A+D \Delta(t) E_{a}$ and $A_{\tau}+D \Delta(t) E_{\tau}$ in (11), respectively. The result then follows from Theorem 10.

\section{Control Design}

On the basis of the previous stability conditions, we will present a design method of robustly stabilizing controllers in this section. For simplicity, we first consider system (6).

Theorem 16. Given scalars $0 \leq h_{1}<h_{2}$ and $\mu$, if there exist scalar $\alpha>0$, positive-definite matrices $P, Q_{i}, i=1,2,3, Z_{j}$, $j=1,2, X$, and matrices $R, S, U$ with appropriate dimensions such that

$$
\Psi=\left[\begin{array}{ccccccccc}
\Psi_{11} & \Psi_{12} & \Psi_{13} & 0 & 0 & 0 & \Psi_{17} & 0 & \Psi_{19} \\
* & \Psi_{22} & X A_{\tau}^{T} & 0 & 0 & h_{2} Z_{1} & -h_{2} X & h_{12} Z_{2} & -h_{12} X \\
* & * & \Psi_{33} & \Psi_{34} & \Psi_{35} & 0 & h_{2} A_{\tau} X & 0 & h_{12} A_{\tau} X \\
* & * & * & \Psi_{44} & 0 & 0 & 0 & 0 & 0 \\
* & * & * & * & \Psi_{55} & 0 & 0 & 0 & 0 \\
* & * & * & * & * & -h_{2} Z_{1} & 0 & 0 & 0 \\
* & * & * & * & * & * & -h_{2} X & 0 & 0 \\
* & * & * & * & * & * & * & -h_{12} Z_{2} & 0 \\
* & * & * & * & * & * & * & * & -h_{12} X
\end{array}\right]
$$

$<0$,

where

$$
\begin{gathered}
\Psi_{11}=A X+X A^{T}+B U+U^{T} B^{T}+\sum_{i=1}^{3} Q_{i}-h_{2}^{-1} E Z_{1} E^{T}, \\
\Psi_{12}=E P+S R^{T}-X+A X+B U, \\
\Psi_{13}=X A_{\tau}^{T}+h_{2}^{-1} E Z_{1} E^{T}, \\
\Psi_{17}=h_{2} A X+h_{2} B U, \quad \Psi_{19}=h_{12} A X+h_{12} B U, \\
\Psi_{22}=-2 X+3 a I, \\
\Psi_{33}=-(1-\mu) Q_{3}-h_{2}^{-1} E Z_{1} E^{T}-h_{12}^{-1} E\left(Z_{1}+Z_{2}\right) E^{T} \\
-h_{12}^{-1} E Z_{2} E^{T}, \\
\Psi_{34}=h_{12}^{-1} E Z_{2} E^{T}, \quad \Psi_{35}=h_{12}^{-1} E\left(Z_{1}+Z_{2}\right) E^{T}, \\
\Psi_{44}=-Q_{1}-h_{12}^{-1} E Z_{2} E^{T}, \\
\Psi_{55}=-Q_{2}-h_{12}^{-1} E\left(Z_{1}+Z_{2}\right) E^{T}
\end{gathered}
$$

and $R_{1} \in \mathbf{R}^{n \times(n-r)}$ is any matrix with full column rank and satisfying $E^{T} R_{1}=0$, then there exists a state feedback controller (7) such that the resulting closed-loop system of system (6) is regular, impulse free, and asymptotically stable. In this case, a suitable controller gain is given by

$$
u(t)=U X^{-1} x(t) .
$$

Proof. With the control law $u(t)=K x(t)$, the resultant closed-loop system of system (6) is

$$
E \dot{x}(t)=(A+B K) x(t)+A_{\tau} x(t-\tau(t)) .
$$

Following the same philosophy as that in [37], we represent system (56) as the following form,

$$
\begin{aligned}
& {\left[\begin{array}{ll}
E & 0 \\
0 & 0
\end{array}\right]\left[\begin{array}{l}
\dot{x}(t) \\
\dot{y}(t)
\end{array}\right]} \\
& \quad=\left[\begin{array}{cc}
0 & I \\
A+B K & -I
\end{array}\right]\left[\begin{array}{l}
x(t) \\
y(t)
\end{array}\right]+\left[\begin{array}{cc}
0 & 0 \\
A_{\tau} & 0
\end{array}\right]\left[\begin{array}{l}
x(t-\tau(t)) \\
y(t-\tau(t))
\end{array}\right],
\end{aligned}
$$

where $y(t)=E \dot{x}(t)$. 
For notational convenience, we introduce

$$
\begin{gathered}
\bar{E}=\left[\begin{array}{ll}
E & 0 \\
0 & 0
\end{array}\right], \quad \bar{A}=\left[\begin{array}{cc}
0 & I \\
A+B K & -I
\end{array}\right], \\
\bar{A}_{\tau}=\left[\begin{array}{cc}
0 & 0 \\
A_{\tau} & 0
\end{array}\right]
\end{gathered}
$$

Then, by Theorem 10, we can show that system (57) is regular, impulse free, and asymptotically stable if (11) holds, where $E$, $A, A_{\tau}, P, Q_{i}$, and $i=1,2,3, Z_{j}, j=1,2, R, S$ are replaced by $\bar{E}, \bar{A}, \bar{A}_{\tau}, \bar{P}, \bar{Q}_{i}, i=1,2,3, \bar{Z}_{j}$, and $j=1,2, \bar{R}, \bar{S}$, respectively. For a special issue, we choose $\bar{P}, \bar{Q}_{i}, \bar{Z}_{j}, \bar{R}$, and $\bar{S}$ as

$$
\begin{aligned}
\bar{P}=\left[\begin{array}{cc}
P & 0 \\
0 & \alpha I
\end{array}\right], & \bar{Q}_{1}=\left[\begin{array}{cc}
Q_{1} & 0 \\
0 & \alpha I
\end{array}\right], \\
\bar{Q}_{2}=\left[\begin{array}{cc}
Q_{2} & 0 \\
0 & \alpha I
\end{array}\right], & \bar{Q}_{3}=\left[\begin{array}{cc}
Q_{3} & 0 \\
0 & \alpha I
\end{array}\right], \\
\bar{Z}_{1}=\left[\begin{array}{cc}
Z_{1} & 0 \\
0 & X
\end{array}\right], & \bar{Z}_{2}=\left[\begin{array}{cc}
Z_{2} & 0 \\
0 & X
\end{array}\right], \\
\bar{R}=\left[\begin{array}{cc}
R & 0 \\
0 & X
\end{array}\right], & \bar{S}=\left[\begin{array}{cc}
S & I \\
0 & I
\end{array}\right],
\end{aligned}
$$

where $P \in \mathbf{R}^{n \times n}, Q_{i} \in \mathbf{R}^{n \times n}, i=1,2,3, Z_{j} \in \mathbf{R}^{n \times n}$, and $j=1,2, X \in \mathbf{R}^{n \times n}$ are symmetrical positive-definite matrices, $R \in \mathbf{R}^{n \times(n-r)}$ is with full column rank and satisfies $E^{\mathrm{T}} R=0$, $S \in \mathbf{R}^{n \times(n-r)}$ is any matrix and $\alpha>0$ is a scalar. It is easy to verify that $\bar{R}$ is with full column rank and satisfies $\bar{E}^{\mathrm{T}} \bar{R}=0$. Then, the following LMI can be obtained:

$$
\left[\begin{array}{ccccccccc}
\Phi_{11} & \Phi_{12} & \Phi_{13} & 0 & 0 & 0 & \Phi_{17} & 0 & \Phi_{19} \\
* & \Phi_{22} & X A_{\tau} & 0 & 0 & h_{2} Z_{1} & -h_{2} X & h_{12} Z_{2} & -h_{12} X \\
* & * & \Phi_{33} & \Phi_{34} & \Phi_{35} & 0 & h_{2} A_{\tau}^{\mathrm{T}} X & 0 & h_{12} A_{\tau}^{\mathrm{T}} X \\
* & * & * & \Phi_{44} & 0 & 0 & 0 & 0 & 0 \\
* & * & * & * & \Phi_{55} & 0 & 0 & 0 & 0 \\
* & * & * & * & * & -h_{2} Z_{1} & 0 & 0 & 0 \\
* & * & * & * & * & * & -h_{2} X & 0 & 0 \\
* & * & * & * & * & * & * & -h_{12} Z_{2} & 0 \\
* & * & * & * & * & * & * & * & -h_{12} X
\end{array}\right]
$$

$<0$,

(60) where

$$
\begin{gathered}
\Phi_{11}=X(A+B K)+(A+B K)^{\mathrm{T}} X+\sum_{i=1}^{3} Q_{i}-h_{2}^{-1} E^{\mathrm{T}} Z_{1} E, \\
\Phi_{12}=E^{\mathrm{T}} P+S_{1} R_{1}^{\mathrm{T}}-X+(A+B K)^{\mathrm{T}} \mathrm{X}, \\
\Phi_{13}=X A_{\tau}+h_{2}^{-1} E^{\mathrm{T}} Z_{1} E, \quad \Phi_{17}=h_{2}(A+B K)^{\mathrm{T}} X, \\
\Phi_{19}=h_{12}(A+B K)^{\mathrm{T}} X, \quad \Phi_{22}=-2 X+3 a I, \\
\Phi_{33}=-(1-\mu) Q_{3}-h_{2}^{-1} E^{\mathrm{T}} Z_{1} E-h_{12}^{-1} E^{\mathrm{T}}\left(Z_{1}+Z_{2}\right) E \\
-h_{12}^{-1} E^{\mathrm{T}} Z_{2} E, \quad \Phi_{34}=h_{12}^{-1} E^{\mathrm{T}}\left(Z_{1}+Z_{2}\right) E, \\
\Phi_{44}^{-1} E^{\mathrm{T}} Z_{2} E, \quad \Phi_{1}-h_{12}^{-1} E^{\mathrm{T}} Z_{2} E, \quad \Phi_{55}=-Q_{2}-h_{12}^{-1} E^{\mathrm{T}}\left(Z_{1}+Z_{2}\right) E .
\end{gathered}
$$

Note that the pairs $(E,(A+B K))$ and $\left(E,\left(A+B K+A_{\tau}\right)\right)$ are regular, causal if and only if the pairs $\left(E^{\mathrm{T}},(A+B K)^{\mathrm{T}}\right)$ and $\left(E^{\mathrm{T}},\left(A+B K+A_{\tau}\right)^{\mathrm{T}}\right)$ are regular, causal. Furthermore, $\operatorname{det}(s E-$ $\left.(A+B K)-e^{-\tau(t) s} A_{\tau}\right)=\operatorname{det}\left(s E^{\mathrm{T}}-(A+B K)^{\mathrm{T}}-e^{-\tau(t) s} A_{\tau}^{\mathrm{T}}\right)$. Then, as long as the regularity, causality, and stability problems are concerned, we can consider the following system instead of (56):

$$
E^{\mathrm{T}} \dot{\zeta}(t)=(A+B K)^{\mathrm{T}} \zeta(t)+A_{\tau}^{\mathrm{T}} \zeta(t-\tau(t))
$$

where $\zeta(t) \in \mathbf{R}^{n}$ is the state vector.

In this sense, (53) can be obtained by replacing $E,(A+$ $B K), A_{\tau}$ in $(60)$ by $E^{\mathrm{T}},(A+B K)^{\mathrm{T}}, A_{\tau}^{\mathrm{T}}$, respectively, and introducing a matrix $U=K X$.

The robust stabilizability result for uncertain singular system (2) is presented in the following theorem.

Theorem 17. Given scalars $0 \leq h_{1}<h_{2}$ and $\mu$, if there exist scalar $\alpha>0$, positive-definite matrices $P, Q_{i}, i=1,2,3, Z_{j}$, $j=1,2, X$, and matrices $R, S, U$ with appropriate dimensions and scalars $\eta_{1}>0, \eta_{2}>0$ such that

$$
\left[\begin{array}{ccccccccccccc}
\Psi_{11} & \Psi_{12} & \Psi_{13} & 0 & 0 & 0 & \Psi_{17} & 0 & \Psi_{19} & \Psi_{110} & \eta_{1} D & \Psi_{112} & 0 \\
* & \Psi_{22} & X A_{\tau}^{T} & 0 & 0 & h_{2} Z_{1} & -h_{2} X & h_{12} Z_{2} & \Psi_{29} & \Psi_{210} & 0 & \Psi_{212} & 0 \\
* & * & \Psi_{33} & \Psi_{34} & \Psi_{35} & 0 & h_{2} A_{\tau} X & 0 & \Psi_{39} & 0 & 0 & 0 & \eta_{2} D \\
* & * & * & \Psi_{44} & 0 & 0 & 0 & 0 & 0 & 0 & 0 & 0 & 0 \\
* & * & * & * & \Psi_{55} & 0 & 0 & 0 & 0 & 0 & 0 & 0 & 0 \\
* & * & * & * & * & -h_{2} Z_{1} & 0 & 0 & 0 & 0 & 0 & 0 & 0 \\
* & * & * & * & * & * & -h_{2} X & 0 & 0 & \Psi_{710} & 0 & \Psi_{712} & 0 \\
* & * & * & * & * & * & * & -h_{12} Z_{2} & 0 & 0 & 0 & 0 & 0 \\
* & * & * & * & * & * & * & * & -h_{12} X & \Psi_{910} & 0 & \Psi_{912} & 0 \\
* & * & * & * & * & * & * & * & * & -\eta_{1} I & \eta_{1} J & 0 & 0 \\
* & * & * & * & * & * & * & * & * & * & -\eta_{1} I & 0 & 0 \\
* & * & * & * & * & * & * & * & * & * & * & -\eta_{2} I & \eta_{2} J \\
* & * & * & * & * & * & * & * & * & * & * & * & -\eta_{2} I
\end{array}\right]<0,
$$


where $\Psi_{11}, \Psi_{12}, \Psi_{13}, \Psi_{17}, \Psi_{19}, \Psi_{22}, \Psi_{33}, \Psi_{34}, \Psi_{35}, \Psi_{44}$, and $\Psi_{55}$ are defined in (53), $\Psi_{29}=-h_{12} X, \Psi_{110}=\Psi_{210}=X E_{a}^{T}+U^{T} E_{b}^{T}$, $\Psi_{112}=\Psi_{212}=X E_{\tau}^{T}, \Psi_{39}=h_{12} A_{\tau} X, \Psi_{710}=h_{2}\left(X E_{a}^{T}+U^{T} E_{b}^{T}\right)$, $\Psi_{910}=h_{12}\left(X E_{a}^{T}+U^{T} E_{b}^{T}\right), \Psi_{712}=h_{2} X E_{\tau}^{T}, \Psi_{912}=h_{12} X E_{\tau}^{T}$, and $R_{1} \in \mathbf{R}^{n \times(n-r)}$ is any matrix with full column rank and satisfying $E^{T} R_{1}=0$; then there exists a state feedback controller (7) such that the resulting closed-loop system of system (2) is regular, impulse free, and robustly asymptotically stable. In this case, a suitable controller gain is given by

$$
u(t)=U X^{-1} x(t)
$$

Proof. Replacing $A$ by $A+D \Delta(t) E_{a}, A_{\tau}$ by $A_{\tau}+D \Delta(t) E_{\tau}$ and $B$ by $B+D \Delta(t) E_{b}$ in (53), respectively, results in the following condition:

$$
\Psi+\Gamma_{1} \Delta(t) \Xi_{1}+\Xi_{1}^{\mathrm{T}} \Delta^{\mathrm{T}}(t) \Gamma_{1}^{\mathrm{T}}+\Gamma_{2} \Delta(t) \Xi_{2}+\Xi_{2}^{\mathrm{T}} \Delta^{\mathrm{T}}(t) \Gamma_{2}^{\mathrm{T}}<0,
$$

where

$$
\begin{gathered}
\Gamma_{1}=\left[\begin{array}{lllllllll}
D^{\mathrm{T}} & 0 & 0 & 0 & 0 & 0 & 0 & 0 & 0
\end{array}\right]^{\mathrm{T}}, \\
\Xi_{1}=\left[\begin{array}{lllllllll}
E_{a} X+E_{b} U & E_{a} X+E_{b} U & 0 & 0 & 0 & 0 & h_{2}\left(E_{a} X+E_{b} U\right) & 0 & h_{12}\left(E_{a} X+E_{b} U\right)
\end{array}\right], \\
\Gamma_{2}=\left[\begin{array}{lllllllll}
0 & 0 & D^{\mathrm{T}} & 0 & 0 & 0 & 0 & 0 & 0
\end{array}\right]^{\mathrm{T}}, \\
\Xi_{2}=\left[\begin{array}{lllllllll}
E_{\tau} U & E_{\tau} U & 0 & 0 & 0 & 0 & h_{2} E_{\tau} X & 0 & h_{12} E_{\tau} X
\end{array}\right],
\end{gathered}
$$

and $\Psi$ is defined in (53).

By Lemma 9, (65) holds for $\Delta(t)$ satisfying (3), if there exist scalars $\varepsilon_{1}>0, \varepsilon_{2}>0$ such that

$$
\begin{aligned}
\Psi & +\left[\begin{array}{ll}
\varepsilon_{1}^{-1} \Xi_{1}^{\mathrm{T}} & \varepsilon_{1} \Gamma_{1}
\end{array}\right]\left[\begin{array}{cc}
I & -J \\
-J^{\mathrm{T}} & I
\end{array}\right]^{-1}\left[\begin{array}{c}
\varepsilon_{1}^{-1} \Xi_{1} \\
\varepsilon_{1} \Gamma_{1}^{\mathrm{T}}
\end{array}\right] \\
& +\left[\begin{array}{ll}
\varepsilon_{2}^{-1} \Xi_{2}^{\mathrm{T}} & \varepsilon_{2} \Gamma_{2}
\end{array}\right]\left[\begin{array}{cc}
I & -J \\
-J^{\mathrm{T}} & I
\end{array}\right]^{-1}\left[\begin{array}{c}
\varepsilon_{2}^{-1} \Xi_{2} \\
\varepsilon_{2} \Gamma_{2}^{\mathrm{T}}
\end{array}\right]<0 .
\end{aligned}
$$

Suppose (63) to be true. Let $\varepsilon_{i}=\sqrt{\eta_{i}}, i=1,2$. Preand postmultiplying the left-hand side matrix of (63) by $\operatorname{diag}\left\{I, I, I, I, I, I, \varepsilon_{1}^{-1}, \varepsilon_{1}^{-1}, \varepsilon_{2}^{-1}, \varepsilon_{2}^{-1}\right\}$ and its transpose, respectively, and using Schur complement equivalence to (63) yields (67). The result then follows from Theorem 16.

\section{Numerical Examples}

In this section, some examples are provided to illustrate the benefits of our results.

Example 1. Consider the nominal unforced part of system (2) with

$$
E=\left[\begin{array}{ll}
1 & 0 \\
0 & 0
\end{array}\right], \quad A=\left[\begin{array}{cc}
0.5 & 0 \\
0 & -1
\end{array}\right], \quad A_{\tau}=\left[\begin{array}{cc}
-1.1 & 1 \\
0 & 0.5
\end{array}\right] .
$$

The case for $h_{1}=0$ and $\mu=0$ has been studied in [12]. We choose $R=\left[\begin{array}{ll}0 & 1\end{array}\right]^{\mathrm{T}}$. The comparison among Corollary 14 in this note and those in $[6-9,11,12,19]$ is listed in Table 1 for $h_{1}=0$. It should be pointed out that the results of [7] fail to deal with the system because the matrix describing the relationship between the fast and slow variables cannot be chosen beforehand. It can be seen that our method is less conservative than those in $[6,9]$ and gives the same results as that in $[8,11,12,19]$. However, when the time delay is a varying delay, our method gives better results than that in [19] for $\mu=0.3$ and $\mu=0.75$ since the relationship among $h_{2}, \tau(t), h_{2}-\tau(t)$, and $\tau(t)-h_{1}$ has been taken into account in Corollary 14. On the other hand, for system with time-varying delay in a range, Table 1 also lists the allowable maximum upper bounds of $h_{2}$ for different $\mu$ with $h_{1}=0.3$ and $h_{1}=0.6$.

Example 2. Consider the unforced part of uncertain system (2) with

$$
\begin{array}{cc}
E=\left[\begin{array}{ll}
1 & 0 \\
0 & 0
\end{array}\right], & A=\left[\begin{array}{cc}
0.4 & 0.2 \\
0 & -1
\end{array}\right], \\
A_{\tau}=\left[\begin{array}{cc}
-1 & 1 \\
0 & 0.3
\end{array}\right], & D=\left[\begin{array}{cc}
0.1 & 0 \\
0 & 0.1
\end{array}\right], \\
E_{a}=\left[\begin{array}{cc}
-0.05 & 0 \\
0 & 0.05
\end{array}\right], & E_{\tau}=\left[\begin{array}{cc}
0.05 & 0 \\
0 & -0.05
\end{array}\right] .
\end{array}
$$

We choose $R=\left[\begin{array}{ll}0 & 1\end{array}\right]^{\mathrm{T}}$. For $h_{1}=0$, the comparison among Theorem 15 in this note and that in [19] is listed in Table 2. It is clear that the result in this note is better than that in [19]. The corresponding maximum upper bounds of $h_{2}$ for different $h_{1}$ and $\mu$ derived by Theorem 15 are also listed in Table 2 .

Example 3. Consider system (2) with

$$
\begin{array}{ll}
E=\left[\begin{array}{ccc}
1 & 1 & 0 \\
1 & -1 & 1 \\
2 & 0 & 1
\end{array}\right], \quad A=\left[\begin{array}{ccc}
1.5 & 0.5 & 1 \\
-1 & 0 & 1 \\
0.5 & 0 & 1
\end{array}\right], \\
A_{\tau}=\left[\begin{array}{ccc}
-1 & 0 & 1 \\
1 & -1 & 0.5 \\
0.3 & 0.5 & -1
\end{array}\right], \quad B=\left[\begin{array}{ll}
1 & 1 \\
1 & 0 \\
0 & 1
\end{array}\right],
\end{array}
$$


TABLE 1: Allowable upper bounds of $h_{2}$ with given $h_{1}$ for different $\mu$.

\begin{tabular}{ccccc}
\hline$h_{1}$ & Methods & $\mu=0$ & $\mu=0.3$ & $\mu=0.75$ \\
\hline & {$[7]$} & - & - & - \\
& {$[9]$} & 0.5567 & - & - \\
0 & {$[6]$} & 0.9680 & - & - \\
& {$[8,11,12]$} & 1.0660 & - & - \\
& {$[19]$} & 1.0660 & 1.0130 & 0.6496 \\
& Corollary 14 & 1.0660 & 1.0263 & 0.7141 \\
\hline 0.3 & Theorem 10 & 1.0660 & 1.0315 & 0.7376 \\
\hline 0.6 & Theorem 10 & 1.0660 & 1.0421 & 0.7426 \\
\hline
\end{tabular}

TABLE 2: Allowable upper bounds of $h_{2}$ with given $h_{1}$ for different $\mu$.

\begin{tabular}{lcccc}
\hline$h_{1}$ & Methods & $\mu=0$ & $\mu=0.3$ & $\mu=0.75$ \\
\hline \multirow{2}{*}{0} & {$[19]$} & 1.1836 & 1.1217 & 1.0307 \\
& Theorem 15 $(J=0)$ & 1.1836 & 1.1348 & 1.1095 \\
\hline \multirow{2}{*}{0.3} & Theorem 15 $(J=0)$ & 1.1836 & 1.1394 & 1.1197 \\
& Theorem 15 $(J=0.1)$ & 1.1842 & 1.1400 & 1.1200 \\
\hline \multirow{2}{*}{0.6} & Theorem 15 $(J=0)$ & 1.1836 & 1.1489 & 1.1317 \\
& Theorem 15 $(J=0.1)$ & 1.1842 & 1.1494 & 1.1321 \\
\hline
\end{tabular}

TABLE 3: Allowable upper bounds of $h_{2}$ with given $h_{1}$.

\begin{tabular}{lcccc}
\hline$h_{1}$ & Methods & $h_{2}$ & $K$ \\
\hline 0 & $\begin{array}{c}\text { Theorem 17 } \\
(J=-0.1)\end{array}$ & 0.9062 & {$\left[\begin{array}{llll}-0.5515 & -0.1800 & -0.7849 \\
-1.0962 & -0.7997 & -0.2503\end{array}\right]$} \\
\hline 0.3 & $\begin{array}{c}\text { Theorem } 17 \\
(J=-0.1)\end{array}$ & \multirow{2}{*}{1.0482} & {$\left[\begin{array}{llll}-0.5845 & -0.1547 & -0.8065 \\
-1.1257 & -0.7953 & -0.2363\end{array}\right]$} \\
\hline 0.6 & $\begin{array}{c}\text { Theorem 17 } \\
(J=-0.1)\end{array}$ & \multirow{2}{*}{1.1919} & {$\left[\begin{array}{llll}-0.6093 & -0.1360 & -0.8218 \\
-1.1582 & -0.7942 & -0.2136\end{array}\right]$} \\
\hline
\end{tabular}

$$
\begin{gathered}
D=\left[\begin{array}{lll}
0.1 & 0.1 & 0.1
\end{array}\right]^{\mathrm{T}}, \quad E_{a}=\left[\begin{array}{lll}
0.2 & 0.1 & 0.3
\end{array}\right], \\
E_{\tau}=\left[\begin{array}{lll}
0.1 & 0.2 & 0.1
\end{array}\right], \quad E_{b}=\left[\begin{array}{ll}
0.1 & 0.1
\end{array}\right], \quad \mu=0.1 .
\end{gathered}
$$

We choose $R=\left[\begin{array}{lll}-1 & 1 & 2\end{array}\right]^{\mathrm{T}}$. According to Theorem 17, Table 3 shows the allowed maximum upper bounds of $h_{2}$ and the corresponding state feedback gain $K$ for different $h_{1}$.

\section{Conclusions}

In this note, the delay-range-dependent robust stability and stabilization for singular time-delay systems with linear fractional uncertainty and time-varying delay in a range are studied. The results are obtained by using the strict LMI approach and constructing an appropriate Lyapunov-Krasovskii functional. Numerical examples have been given to demonstrate the effectiveness of the presented criteria and their improvement over existing results.

\section{Acknowledgments}

This work was supported by the opening Foundation of the Key Laboratory of Measurement and Control of Complex
Systems of Engineering, Ministry of Education, Southeast University (MCCSE2012A06), and the Scientific Research Foundation of Nanjing University of Posts and Telecommunications (NY210080).

\section{References}

[1] J. K. Hale and S. M. Verduyn Lunel, Introduction to Functional Differential Equations, Springer, New York, NY, USA, 1993.

[2] V. B. Kolmanovskii and V. R. Nosov, Stability of Functional Differential Equations, Academic, New York, NY, USA, 1986.

[3] S. Y. Xu, P. Van Dooren, R. Ştefan, and J. Lam, "Robust stability and stabilization for singular systems with state delay and parameter uncertainty," IEEE Transactions on Automatic Control, vol. 47, no. 7, pp. 1122-1128, 2002.

[4] S. S. Zhou and J. Lam, "Robust stabilization of delayed singular systems with linear fractional parametric uncertainties," Circuits, Systems, and Signal Processing, vol. 22, no. 6, pp. 579-588, 2003.

[5] S. Xu, J. Lam, and L. Zhang, "Robust D-stability analysis for uncertain discrete singular systems with state delay," IEEE Transactions on Circuits and Systems I, vol. 49, no. 4, pp. 551$555,2002$.

[6] E. Fridman and U. Shaked, " $H_{\infty}$ control of linear state-delay descriptor systems: an LMI approach," Linear Algebra and Its Applications, vol. 351-352, pp. 271-302, 2002.

[7] E. K. Boukas and Z. K. Liu, "Delay-dependent stability analysis of singular linear continuous-time system," IEE Proceedings, vol. 150, no. 4, pp. 325-330, 2003.

[8] H. Su, X. Ji, and J. Chu, "Delay-dependent robust control for uncertain singular time-delay systems," Asian Journal of Control, vol. 8, no. 2, pp. 180-189, 2006.

[9] R. Zhong and Z. Yang, "Delay-dependent robust control of descriptor systems with time delay," Asian Journal of Control, vol. 8, no. 1, pp. 36-44, 2006.

[10] Z. P. Wu and W. Zhou, "Delay-dependent robust $H_{\infty}$ control for uncertain singular time-delay systems," IET Control Theory and Applications, vol. 1, no. 5, pp. 1234-1241, 2007.

[11] S. Zhu, C. Zhang, Z. Cheng, and J. Feng, "Delay-dependent robust stability criteria for two classes of uncertain singular time-delay systems," IEEE Transactions on Automatic Control, vol. 52, no. 5, pp. 880-885, 2007.

[12] Z. Wu and W. Zhou, "Delay-dependent robust stabilization for uncertain singular systems with state delay," Acta Automatica Sinica, vol. 33, no. 7, pp. 714-718, 2007.

[13] M. Fang, "Delay-dependent robust $H_{\infty}$ control for uncertain singular systems with state delay," Acta Automatica Sinica, vol. 35, no. 1, pp. 65-70, 2009.

[14] Z. Wu, H. Su, and J. Chu, "Improved results on delay-dependent $H_{\infty}$ control for singular time-delay systems," Acta Automatica Sinica, vol. 35, no. 8, pp. 1101-1106, 2009.

[15] Y.-F. Feng, X.-L. Zhu, and Q.-L. Zhang, "Delay-dependent stability criteria for singular time-delay systems," Acta Automatica Sinica, vol. 36, no. 3, pp. 433-437, 2010.

[16] S. Zhu, Z. Li, and C. Zhang, "Delay decomposition approach to delay-dependent stability for singular time-delay systems," IET Control Theory and Applications, vol. 4, no. 11, pp. 2613-2620, 2010.

[17] Z. Feng, J. Lam, and H. Gao, " $\alpha$-Dissipativity analysis of singular time-delay systems," Automatica, vol. 47, no. 11, pp. 2548-2552, 2011. 
[18] D. Yue and Q.-L. Han, "A delay-dependent stability criterion of neutral systems and its application to a partial element equivalent circuit model," IEEE Transactions on Circuits and Systems II, vol. 51, no. 12, pp. 685-689, 2004.

[19] H. Wang, A. Xue, R. Lu, and J. Wang, "Delay-dependent robust stability and stabilization for uncertain singular system with time-varying delay," in Proceedings of the American Control Conference, pp. 1327-1331, Washington, DC, USA, 2008.

[20] A. Haidar and E. K. Boukas, "Exponential stability of singular systems with multiple time-varying delays," Automatica, vol. 45, no. 2, pp. 539-545, 2009.

[21] Z. Wu, H. Su, and J. Chu, "Delay-dependent $H_{\infty}$ filtering for singular Markovian jump time-delay systems," Signal Processing, vol. 90, no. 6, pp. 1815-1824, 2010.

[22] Z. Wu, H. Su, and J. Chu, " $H_{\infty}$ filtering for singular systems with time-varying delay," International Journal of Robust and Nonlinear Control, vol. 20, no. 11, pp. 1269-1284, 2010.

[23] J. Lin and S. Fei, "Reliable control for a class of uncertain singular systems with interval time-varying delay," Asian Journal of Control, vol. 13, no. 4, pp. 542-552, 2011.

[24] X. Zhu, Y. Wang, and Y. Gan, " $H_{\infty}$ filtering for continuous-time singular systems with time-varying delay," International Journal of Adaptive Control and Signal Processing, vol. 25, no. 2, pp. 137154, 2011.

[25] L. Chen, M.-Y. Zhong, and M.-Y. Zhang, " $H_{\infty}$ fault detection for linear singular systems with time-varying delay," International Journal of Control, Automation and Systems, vol. 9, no. 1, pp. 914,2011

[26] F. Li and X. Zhang, "A delay-dependent bounded real lemma for singular LPV systems with time-variant delay," International Journal of Robust and Nonlinear Control, vol. 22, no. 5, pp. 559574,2012 .

[27] Y. Ding, H. Zhu, S. Zhong, and Y. Zeng, "Exponential meansquare stability of time-delay singular systems with Markovian switching and nonlinear perturbations," Applied Mathematics and Computation, vol. 219, no. 4, pp. 2350-2359, 2012.

[28] T. Li, L. Guo, and Y. Zhang, "Delay-range-dependent robust stability and stabilization for uncertain systems with time-varying delay," International Journal of Robust and Nonlinear Control, vol. 18, no. 13, pp. 1372-1387, 2008.

[29] Y. Xia, E. Boukas, P. Shi, and J. Zhang, "Stability and stabilization of continuous-time singular hybrid systems," Automatica, vol. 45, no. 6, pp. 1504-1509, 2009.

[30] G. I. Bara, "Robust analysis and control of parameter-dependent uncertain descriptor systems," Systems and Control Letters, vol. 60, no. 5, pp. 356-364, 2011.

[31] S. Boyd, L. E. Ghaoui, E. Feron, and V. Balakrishnan, Linear Matrix Inequalities in System and Control Theory, SIAM, Pennsylvania, Pa, USA, 1994.

[32] L. Xie, "Output feedback $H_{\infty}$ control of systems with parameter uncertainty," International Journal of Control, vol. 63, no. 4, pp. 741-750, 1996.

[33] L. Dai, Singular Control Systems, Springer, New York, NY, USA, 1989.

[34] Q.-L. Han and D. Yue, "Absolute stability of Lur'e systems with time-varying delay," IET Control Theory and Applications, vol. 1, no. 3, pp. 854-859, 2007.

[35] M. Krstic and H. Deng, Stabilization of Nonlinear Uncertain Systems, Springer, London, UK, 1998.
[36] X.-M. Zhang and Q.-L. Han, "A delay decomposition approach to delay-dependent stability for linear systems with timevarying delays," International Journal of Robust and Nonlinear Control, vol. 19, no. 17, pp. 1922-1930, 2009.

[37] E. Fridman and U. Shaked, "A descriptor system approach to $H_{\infty}$ control of linear time-delay systems," IEEE Transactions on Automatic Control, vol. 47, no. 2, pp. 253-270, 2002. 


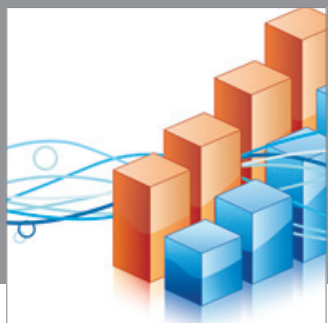

Advances in

Operations Research

mansans

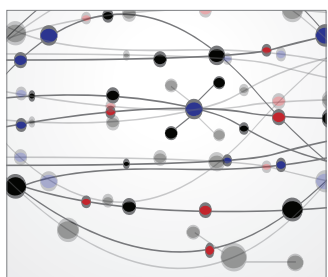

The Scientific World Journal
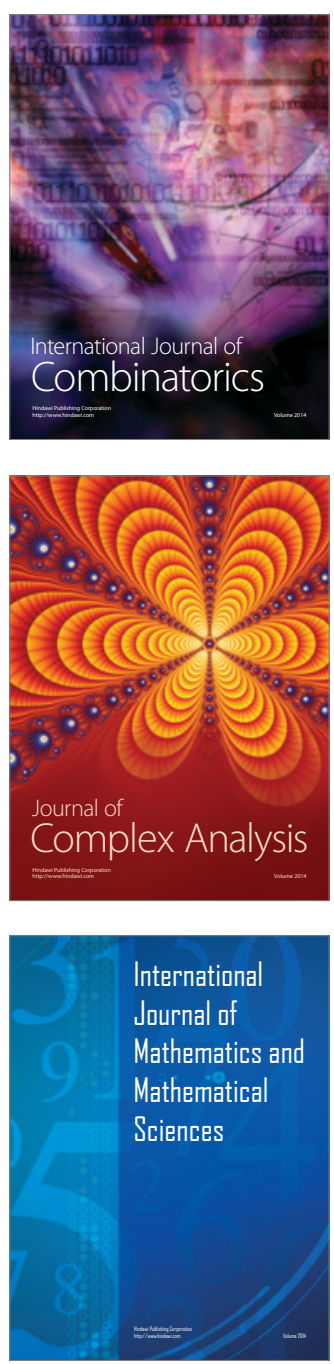
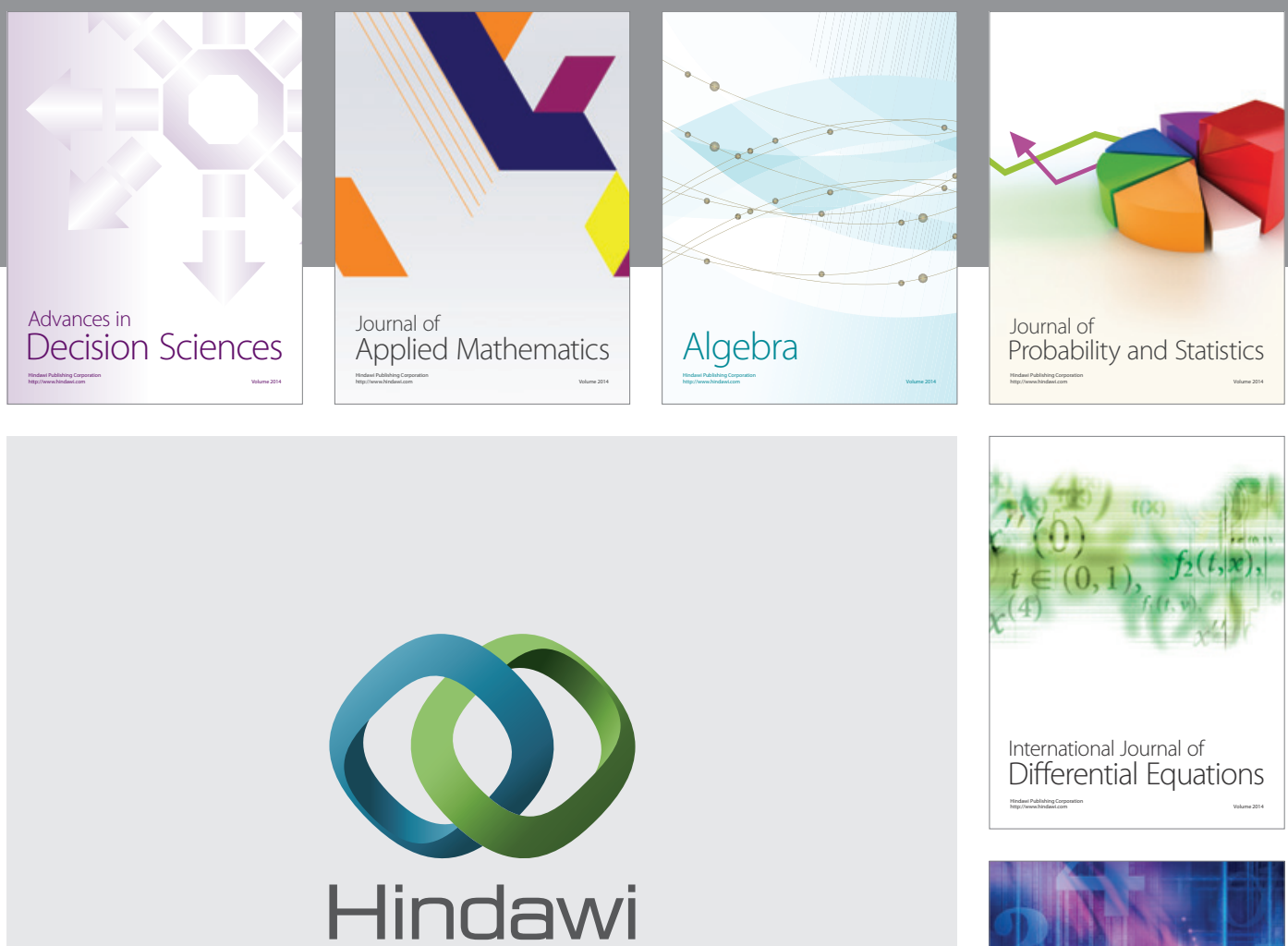

Submit your manuscripts at http://www.hindawi.com
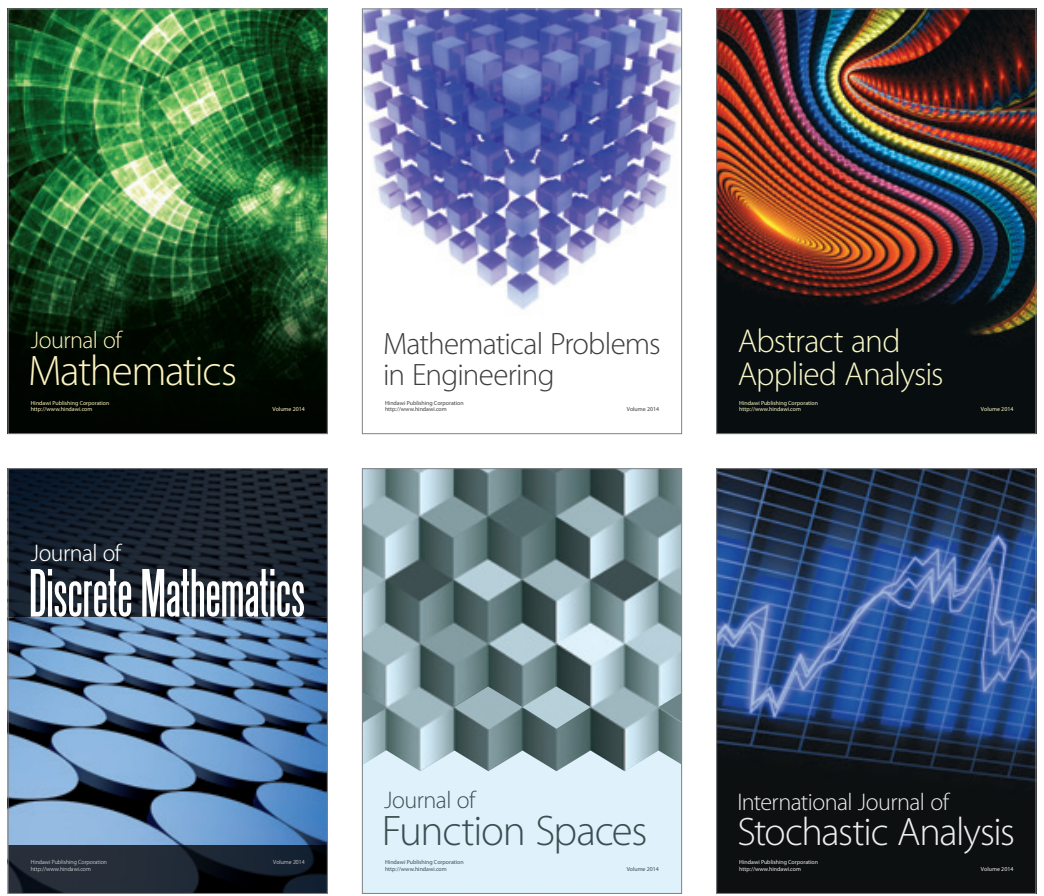

Journal of

Function Spaces

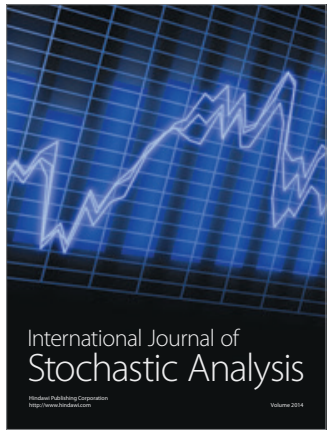

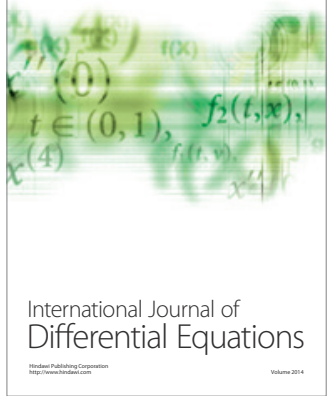
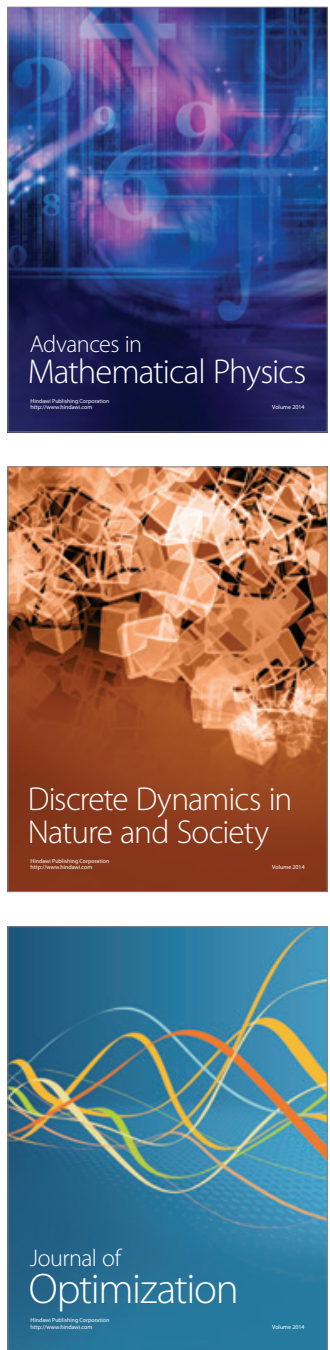